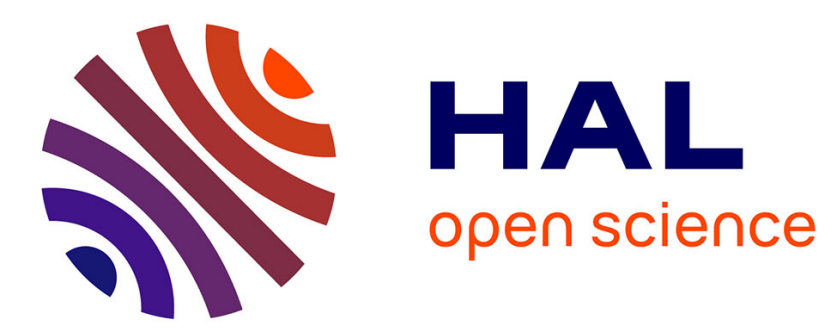

\title{
INVESTIGATION OF HOT CARRIER RELAXATION WITH PICOSECOND LASER PULSES
}

\author{
J. Shah
}

\section{To cite this version:}

J. Shah. INVESTIGATION OF HOT CARRIER RELAXATION WITH PICOSEC-

OND LASER PULSES. Journal de Physique Colloques, 1981, 42 (C7), pp.C7-445-C7-462. 10.1051/jphyscol:1981755 . jpa-00221692

\section{HAL Id: jpa-00221692 https://hal.science/jpa-00221692}

Submitted on 1 Jan 1981

HAL is a multi-disciplinary open access archive for the deposit and dissemination of scientific research documents, whether they are published or not. The documents may come from teaching and research institutions in France or abroad, or from public or private research centers.
L'archive ouverte pluridisciplinaire HAL, est destinée au dépôt et à la diffusion de documents scientifiques de niveau recherche, publiés ou non, émanant des établissements d'enseignement et de recherche français ou étrangers, des laboratoires publics ou privés. 


\section{INVESTIGATION OF HOT CARRIER RELAXATION WITH PICOSECOND LASER PULSES}

\section{J. Shah}

BeII Laboratories, Holmdel, N.J., 07733, U.S.A.

RÉsume - La disponibilité récente de lasers sous-picoseconde a augmenté l'utilisation de techniques optiques pour l'étude de la relaxation de porteurs chauds dans les semiconducteurs.

Dans cet article, nous présenterons d'abord des concepts théoriques de base associés à l'étude à la picoseconde de la relaxation des porteurs.

Nous exposerons ensuite des résultats expérimentaux sur la relaxation des porteurs chauds dans l'AsGa à la suite d'une excitation par un laser sous-picoseconde. Les temps de relaxation observés seront comparés à la théorie et on mettra en évidence l'écrantage de l'interaction electron-phonon.

Enfin deux expériences récentes combinant des techniques de transport et d'optique à la picoseconde seront aiscutées pour illustrer leur utilisation et les possibilités des impulsions laser à la picoseconde dans l'étude des effets des porteurs chauds dans les semiconducteurs.

Abstract - Recent availability of subpicosecond lasers has enhanced the usefulness of optical techniques in the investigation of hot carrier relaxation in semiconductors by allowing us to directly probe the carrier distribution function on picosecond timescales. In this review, we will first discuss some basic theoretical concepts related to picosecond investigation of carrier relaxation. We will then review recent experimental results on hot carrier relaxation in GaAs following excitation by a subpicosecond laser. observed relaxation rates will be compared with theory and evidence for the screening of electron-phonon interaction will be presented. Finally two recent experiments combining transport and picosecond optical techniques will be discussed to further illustrate the utility and possibilities of picosecond laser pulses in the investigation of hot carrier effects in semiconductors.

1. Introduction. - Physics of hot carriers deals with the many complex and interesting problems relating to energy and momentum exchange between electrons and holes, between carriers and phonons, and between carriers and impurities or imperfections. An understanding of these interactions is important not only from a fundamental viewpoint but also for modelling the performance of semiconductor devices, particularly those based on the transport of charge carriers at low and high electric fields. Early impetus for the work on hot electron physics was provided by devices such as Gunn oscillators which depend for their operation on the transferred electron effect. With the present trend towards smaller and faster devices, and the emergence of VLSI technology, the study of nonlinear electronic transport and hot electron physics remains an active area of research.

Classical transport measurements have contributed much to our understanding of various carrier scattering processes and have 
also provided quantitative information about the average energy and momentum scattering rates for these processes. (1) optical measurements complement these transport measurements in many respects. While transport measurements provide a measure of the average carrier response to an applied electric field, optical measurements offer the best means of determining the electron and hole distribution functions (DF) and the microscopic (i.e., not averaged over a DF) scattering rates so important for a theoretical description of the transport processes. Various sensitive and high energy resolution optical techniques such as transmission, reflection, emission and Raman spectroscopies have been used in the past to probe these aspects of the carrier system under various experimental conditions. Some of these techniques have been discussed in recent reviews. $(2,3)$

optical techniques have been applied to the hot electron field in another way also. It has been shown that even in the absence of an external electric field, optical techniques can be used to create a hot, nonequilibrium carrier distribution. $(4,5)$ Further, the heating of carriers by photoexcitation can be controlled by controlling such parameters as the excitation intensity $\left(I_{X}\right)$ and/or the excitation photon energy $\left(E_{x}\right) \cdot(4,5,6)$ In contrast to the case of electric field induced heating, photoinduced heating is found ${ }^{(4)}$ to generally produce a Maxwellian DF, although non-Maxwellian DF have been produced under extreme conditions. (7) While the mechanism of generating hot carriers by photoexcitation is quite different from heating by an electric field, the same carrier relaxation and scattering processes apply in both cases. Thus the ability to create and probe hot carriers by optical techniques has become a powerful means of studying the processes important in hot electron physics. $(8,9)$ The recent development $(10)$ of picosecond and subpicosecond lasers have immensely increased the value of these optical techniques by extending the timescale on which meaningful measurements can be made into a range not accessible as yet to purely electrical methods. Since many of the scattering processes important to carrier dynamics occur on this time scale, one has the ability, in principle, to determine directly these scattering rates and even to monitor the time evolution of $D F$ at very short times. In this review we will discuss the recent work applying the picosecond optical techniques to III-V semiconductors of which GaAs is a prototype. (II) Many exciting results have already been obtained. A direct measurement of carrier cooling rate has been made. $(12,13,14)$ The importance of the screening of electron-phonon interaction has been established. (13) 
Carrier relaxation in two-dimensional quantum wells (15) and velocity overshoot of electrons in a high electric field ${ }^{(16)}$ have been investigated. Super high-density $\left(\geq 10^{20} \mathrm{~cm}^{-3}\right)$ plasma has been generated (17) by using picosecond pulses and studied by observing the resulting short-pulse semiconductor laser dynamics. These many varied results make it clear that picosecond lasers provide a means of determining many parameters which are important in hot electron physics and whose knowledge is essential in understanding the smaller and faster semiconductor devices of today and tomorrow. In this review, we will first discuss some important theoretical considerations (Sec. II), and then discuss recent experimental work in this field (Sec. III).

II. Theoretical Considerations

II. A photoinduced Heating of Carriers

The absorption of photons by a semiconductor generates a nonequilibrium carrier distribution in the conduction and/or valence bands. (18) These nonequilibrium carriers relax extremely rapidly (< lpsec) to the band extrema by emitting phonons and plasmons, and by interacting with other carriers. Since the typical carrier lifetimes are at least a couple of orders of magnitude larger than these relaxation times, the density of the system of carriers near the band extrema is much larger than the density of the relaxing photoexcited carriers, except at times immediately following the excitation by a subpicosecond laser pulse. Let us first consider the case of CW excitation (defined here as any experiment with time resolution larger than $1 \mathrm{nsec}$ ). The photoexcited electrons generated with an excess energy $\Delta \mathrm{E}_{e}$ (Fig. l) exchange a fraction $f$ of this energy with the carriers at the bottom of the band through carrier-carrier scattering; the remaining energy is given to the lattice by the emission of (mostly) optical phonons. The fraction $f$ depends on many para-
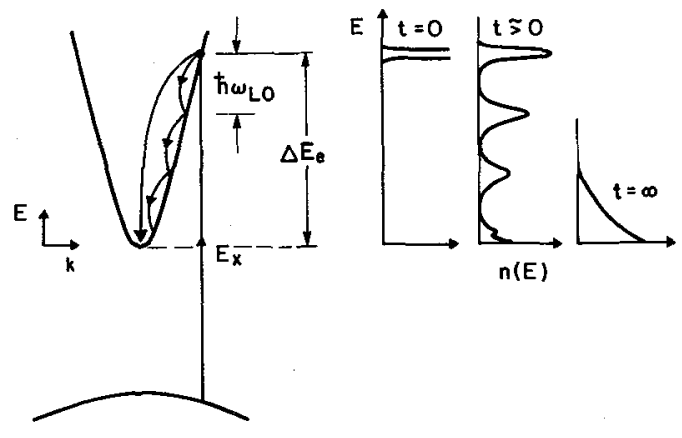

Fig. 1 Schematic diagram showing (left) relaxation processes following interband absorption and (right) carrier DF at three time delays 
meters including $\Delta \mathrm{E}_{e}$, the carrier density, and the intercarrier and the carrier-phonon energy exchange rates. The carrier system loses this excess energy to the lattice also by emission of phonons. If the rate of energy input to the carriex system is sufficiently large, the average energy of the carriers becomes measurably larger than $\frac{3}{2} \mathrm{kT}_{L}$ where $\mathrm{T}_{\mathrm{I}}$ is the lattice temperature. The relative values of various energy exchange rates and the carrier lifetimes determine whether the carriers can be described by an equilibrium (MaxwellBoltzmann or Fermi-Dirne) DF with a carrier temperature $T_{C}>{ }^{T}$ or a nonequilibrium distribution for which a temperature cannot be defined. (4)

Photoexcitation by picosecond lasers involves somewhat different considerations. One asks the question: how does a monoenergetic carrier distribution created by an ultrashort light pulse evolve with time. Optical pulses shorter than 0.1 psec have recently become available. (19) Since this is comparable to the phonon emission time by an electron with $\sim 0.1 \mathrm{eV}$ energy in GaAs, an electron cascading down the conduction band by emitting Lo phonons (Fig. 1) may take several pulse widths to reach an energy less than hil ${ }_{L O}$ (the phonon energy). During this time, an appropriate description of the carrier-carrier interactions is difficult to define. However, the cascading electrons can be expected to lose energy by collective excitations (plasmons) of the photoexcited carrier system and direct carrier-carrier collisions. Both these processes depend on electron densitios. Therefore, depending on the photoexited carrier densities and other material and photoexcitation parameters, the DF may evolve in a complicated manner for times of the order of $1 \mathrm{psec}$, as illustrated schematically in Fig. $1(t>0)$. At longer times, a steady state similar to that obtained for $C W$ excitation emerges and the $D F$ becomes a Maxwellian for sufficiently high carrier densities as illustrated in Fig. $1(t=\infty)$.

While these concepts are simple in principle, several complications must be considered for a realistic semiconductor. In case of GaAs, the presence of light and heavy valence bands and their warping creates complications. The presence of screening of various interactions at high carrier densities, nonlinear effects (e.g., large disturbance in the phonon population generated by cascading electrons) and nonuniform carrier densities must be considered. Detailed theoretical calculations taking account of these effects. would be extremely useful both for the $\mathrm{CW}$ and subpicosecond excitations. 


\section{II.B High Density Plasma in a Semiconductor}

Picosecond lasers provide a unique technique of exciting a high-density plasma without damaging the semiconductor; densities in excess of $10^{20}$ electron-hole pairs $/ \mathrm{cm}^{3}$ can easily be achieved. (17) The presence of a dense plasma modifies the properties of a semiconductor, and an understanding of these modifications is essential for the interpretation of experiments. We therefore present a brief review of our present understanding in this field.

At low temperatures and in the absence of carriers, the optical absorption spectrum of intrinsic GaAs consists of one (or more) sharp exciton absorption lines at energies slightly below the bandgap energy $E_{g}$, followed by a slowly rising continuum above $E_{g}$ (Fig. 2a). The sharp lines are due to hydrogen-like bound states of

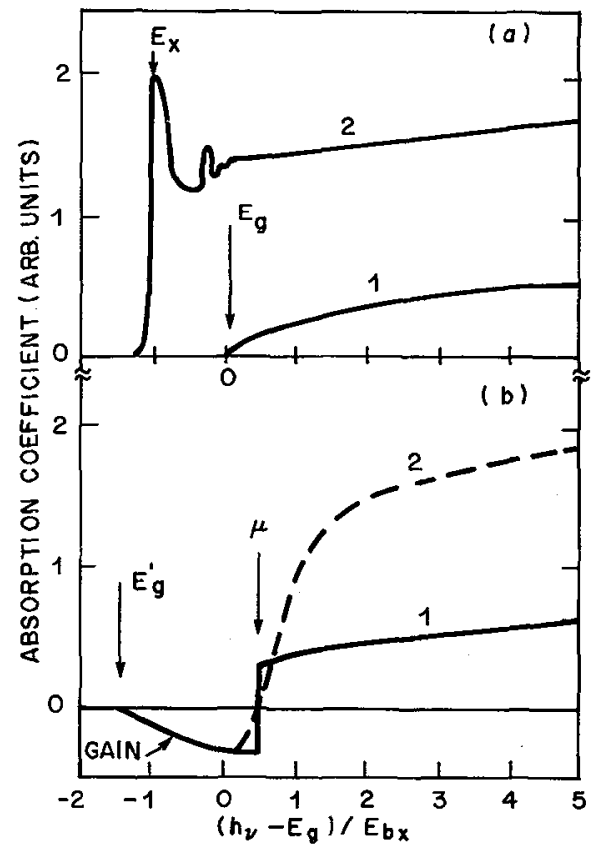

Fig. 2 Schematic diagram of the low temperature absorption coefficient of a direct gap semiconductor like GaAs. (a) without and (b) with photoexcited carriers. Curves 2 include excitonic effects which are neglected in curves 1 .

excitons while the continuum is due to interband absorption considerably enhanced by excitonic contributions. With the excitation of a high density plasma in GaAs, the discrete exciton states are screened and the enhancement of the absorption coefficient a above $\mathrm{E}_{\mathrm{g}}$ is reduced for $\left(\mathrm{h} \nu-\mathrm{E}_{\mathrm{g}}\right)$ less than and comparable to $\hbar \omega_{p}$ where $\omega_{p}$ is the plasma frequency. However, the enhancement for ( $h \nu-E_{g}$ ) >> $h \omega_{p}$ is not affected. (20) In addition, the exchange and correlation effects in the electron-hole plasma result in a renormalization (reduction) of the bandgap. $(21,22)$ similar phenomena are responsible 
for the creation of electron-hole droplets in materials such as Ge and $\mathrm{Si}$, and electron-hole liquids in materials such as GaP ${ }^{(23)}$ and cds. (24) Experimentally important parameters, the renormalized bandgap energy $E_{g}^{\prime}$ and the chemical potential $\mu\left(=E_{g}^{\prime}+\right.$ the sum of the electron and hole quasi-Fermi energies), can be deduced from the theoretical calculation of exchange and correlation energies. Results of such a calculation $(20)$ for GaAs are shown in Fig. 3. One

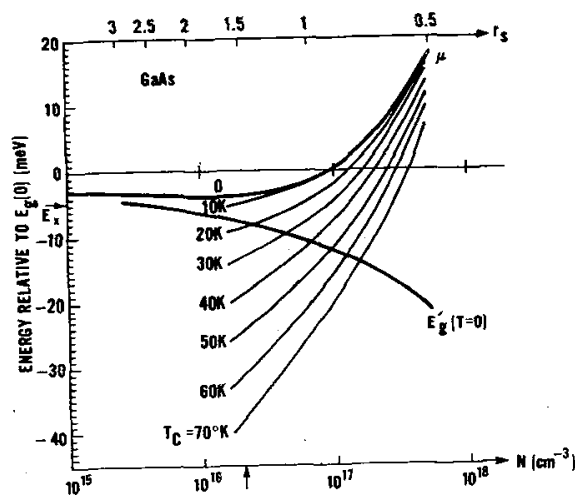

Fig. 3 Calculation of the renormalized bandgap energy $\left(E_{q}^{\prime}\right)$ and the chemical potential ( $\mu$ ) in GaAs as a function of density at various temperatures (from Shah et al., Ref. 20).

expects optical gain for $E_{g}^{\prime}<h \nu<\mu$ and optical absorption for hv > $\mu$. At $\mathrm{T}_{\mathrm{L}}=0$, the transition from gain to absorption is sharp; at finite $T$, the carriers also occupy states above $\mu$ and affect $\alpha$ for h $\nu>\mu$. These screening, renormalization and band-filling effects lead to changes in the absorption spectrum as illustrated in Fig. 2b. Analysis of a measured absorption spectra at various delays including these effects can, in principle, allow one to determine the DF as a function of time. Similar information can also be obtained from band-to-band luminescence experiments providing these plasma effects are accounted for properly.

The simplified picture described above has to be modified in several respects in a realistic case. The difficulties associated with deducing a DF from the measured absorption spectrum have been discussed earlier. $(2,3)$ The theories of exchange and correlation energy for high density plasma in semiconductors have only been developed for $T=0$ and may have to be modified drastically at high carrier temperatures or for the non-Maxwellian DF expected in picosecond measurements. Auger recombination, plasma expansion, etc., also need to be considered. A great deal more theoretical work is required to properly account for these effects.

II.C Cooling of a Hot Carrier Distribution

We are interested in calculating the average energy loss rate per electron-hole pair $\left\langle\frac{d E}{d t}\right\rangle=P_{C}$ for a Maxwellian DF of 
electrons and holes which are at a temperature $T_{C}$ greater than the lattice temperature $\mathrm{T}_{\mathrm{L}}$. For electrons, $\mathrm{P}_{\mathrm{C}}$ is dominated by polar optical phonons at high $\mathrm{T}_{\mathrm{C}}$ and acoustic phonons $\mathrm{T}_{\mathrm{C}}<30 \mathrm{k}$. Holes exhibit similar behaviour, however, the hole-acoustic phonon coupling is much larger because of the heavy mass of holes in GaAs. For this reason $P_{C}$ is completely dominated by holes for $T_{C}<40 \mathrm{k}$. At higher temperatures where interactions with optical phonons dominate, both polar and nonpolar interactions are important for the holes because of their p-like wave functions. (25) Goebel and Hildebrand (26) have considered these factors in detail. Using $\mathrm{m}_{e}=0.07 \mathrm{~m}_{0}, \mathrm{~m}_{h}=0.62 \mathrm{~m}$, and other values of parameters same as by Goebel and Hildebrand, we obtain the curve shown in Fig. 4 for. $P_{C}$ as a function of $T_{C}$. The actual values will be somewhat $(\approx 50 \%)$ higher when nonpolar scattering of holes is included. The rate $\left\langle\frac{d T}{d t}\right\rangle$ is $\left(P_{C} / 3 k\right)$, and is also shown in Fig. 4. One can now calculate $\mathrm{T}_{\mathbf{C}}(t)$ simply by

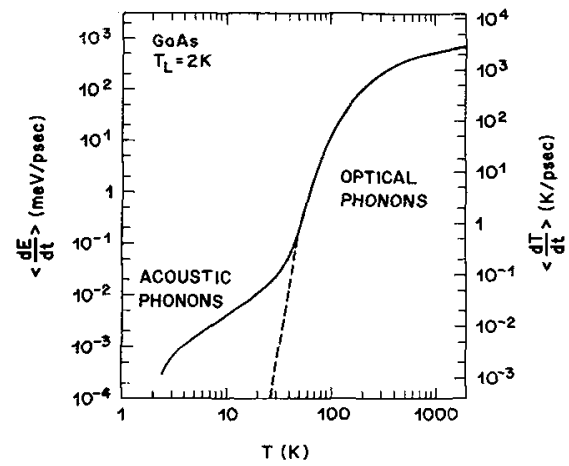

Fig. 4 Calculated average energy loss rate per electron-hole pair for a Maxwellian plasma at temperature $T$ in GaAs (after Goebel and Hildebrand, Ref. 26).

integrating this curve for a given initial temperature $T_{0}=T_{c}(0)$. Some examples are shown in Fig. 5. We have introduced an adjustable carrier-phonon coupling parameter $\mathrm{C}$ into the calculations. $\mathrm{C}=1$. corresponds to the curve shown in Fig. 4; thus effective $\mathrm{P}_{\mathrm{C}}=\mathrm{C} \mathrm{x}$ ( $P_{C}$ shown in Fig. 4) can be defined to take account of the change in carrier-phonon interaction due to screening and other effects. We note that the rate of cooling slows down with time and becomes extremely slow when $\mathrm{T}_{\mathrm{C}}<40 \mathrm{k}$ where acoustic phonons dominate. For a typical carrier lifetime of $10^{-9} \mathrm{sec}$, the carriers are likely to recombine before reaching a lattice temperature $\mathrm{T}_{\mathrm{L}}=2 \mathrm{k}$.

\section{Experimental Results}

Several techniques for generating picosecond and subpicosecond optical pulses have been developed in recent years. (10) The use of these picosecond sources in studying hot carrier relaxation 


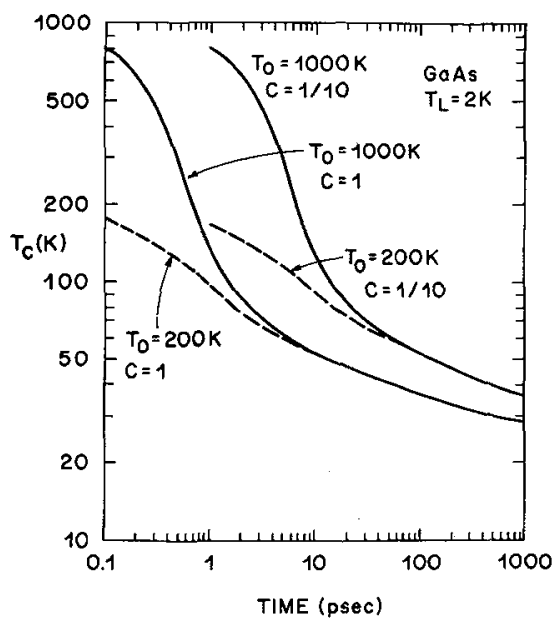

Fig. 5 Calculated variation of the carrier temperature $\mathrm{T}_{\mathrm{C}}$ as a function of time delay for the initial temperature $T_{0}=1000 \mathrm{~K}$, and $\mathrm{T}_{0}=200 \mathrm{~K}$, and $\mathrm{C}^{0}=1$ and $\mathrm{I} / 10$.

in semiconductors can be best illustrated by discussing some recent experiments performed by using these techniques. We first discuss a set of experiments performed on GaAs at low temperatures by Shank et al (12) and teheny et al. (13) A passively mode-locked dye laser (pulsewidth 0.5 psec) was cavity dumped and amplified in a three-stage amplifier pumped by a frequency doubled YAG laser. (27) The amplified beam was split into two beams; one beam was focused on a cell containing ethanol to generate a Raman shifted beam at $1.65 \mathrm{ev}$ which photoexcited the crystal of GaAs. Changes in the absorption spectrum of the photoexcited GaAs were monitored with broadband subpicosecond continuum generated by focusing the second amplified beam into a cell containing water. (27) A controlled time delay between the pump and probe pulses was introduced by varying the optical path length of the probe beam. For a fixed delay, the entire absorption spectrum in the region of interest could be recorded for every pulse and averaged over a large number of pulses by using an optical multichannel analyzer (OMA). Alternatively, the time evolution of absorption at a given photon energy could be measured by varying the optical delay with a stepper motor while recording the signal from a single channel of OMA into a signal averager.

The transmission spectrum of GaAs obtained with such a picosecond continuum, in the absence of pump pulses $(\tau<0)$, is shown in Fig. 6a. This represents the unperturbed transmission spectrum obtained when no photoexcited carriers are present. It shows high transmission below the bandgap ( $\lambda>8150 \AA$ ), a sharp dip at exciton energy and a slowly decreasing transmission at shorter 

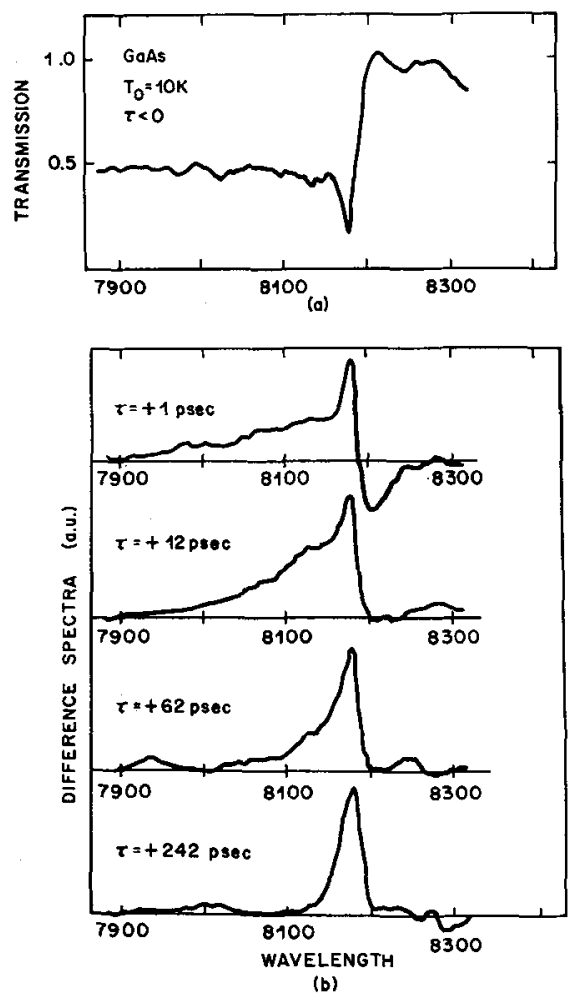

Fig. 6 (a) Transmission spectrum of GaAs obtained with 0.5 psec broadband continuum pulses without any photoexcitation. (b) Difference spectra at various time delays following excitation by a $0.5 \mathrm{psec}$ pump pulse (from Leheny et al., Ref. 13)

-wavelengths (compare with curve 2 of Fig. 2a). In Fig. $6 \mathrm{~b}$ we present the difference spectra at various time delays ( $\tau=1 \mathrm{psec}$ to 242 psec) obtained by subtracting the unperturbed spectrum of $\mathrm{Fig}$. $6 \mathrm{a}$ from the measured spectrum at a given delay $\tau$; (i.e., a positive signal implies an increase in transmission). The important feature of the data are asfollows: For hv $<\mathrm{E}_{\mathrm{g}}$, there is a decrease in transmission at 1 psec which was attributed to the shift in the absorption edge due to the renormalization of the bandgap (Section II B). This decrease in transmission does not persist beyond $\approx 12$ psec because the photoexcited carriers fill the states between the renormalized bandgap $\left(E_{g}^{\prime}\right)$ and the unperturbed bandgap $E_{g}$. For sufficiently high excitation density, one observes gain between $\mathrm{E}_{g}^{\prime}$ and the chemical potential $\mu$ (see Fig. 2b). The second feature of the data is a sharp increase in transmission at $\mathrm{E}_{\mathrm{x}}$, attributed to the screening of exciton bound states. A remarkable feature of the data is that the screening and renormalization are effective in < 1 psec while the majority of carriers occupy states high in the band and persist for at least 
242 psec. The third important feature of the data is the increase in transmission at shorter wavelengths ( $h v>E_{g}$ ) which was attributed to changes in the Fermi occupation factors or the distribution function DF. Each of these features represent the observation of important changes in the characteristics of GaAs on a time scale never before accessible, but we will restrict our discussion here to the analysis of this last feature which is the one of most interest for determining hot carrier relaxation rates in GaAs.

To obtain more quantitative information about carrier relaxation rates, the measured transmission spectra at various delays were fitted using the methods previously applied to the analysis of the steady state experiment. ${ }^{(20)}$ The carrier density $(n)$ and temperature $\mathrm{T}_{\mathbf{c}}$ were treated as adjustable parameters. The fit for the spectra measured at $\tau=10 \mathrm{psec}$ for three different excitation intensities $I_{x}$ are shown in Fig. 7. At the highest $I_{x}$ (curve (c)), there

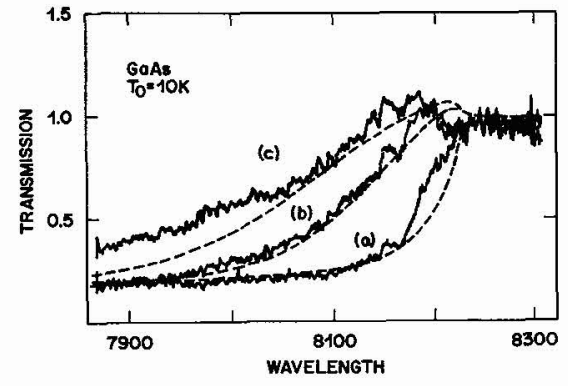

Fig. 7 Transmission spectrum of GaAs at 10 psec following photoexcitation at three pump intensities $\mathrm{I}_{0}, 4 \mathrm{I}_{0}$ and $15 \mathrm{I}_{0}$ with $\mathrm{I}_{0}=$ $2 \times 10^{7} \mathrm{~W} / \mathrm{cm}^{2}$. Dashed curves are theoretical fits as described in the text for (a) $\mathrm{n}=7 \times 1016 \mathrm{~cm}^{-3}$, $\mathrm{T}_{\mathrm{C}}=50 \mathrm{~K}(\mathrm{~b}) \mathrm{n}=3 \times 10^{17} \mathrm{~cm}^{-3}$ and $\mathrm{T}_{\mathrm{C}}=90 \mathrm{~K}$ and $(\mathrm{c}) \mathrm{n}=1 \times 10^{18} \mathrm{~cm}^{-3}$ and $\mathrm{T}_{\mathrm{C}}=140 \mathrm{~K}$ (from Leheny et al., Ref. (3).

is considerably larger population at shorter wavelengths than predicted by Maxwelliam DF. Similar nonequilibrium distributions have also been inferred from $\mathrm{CW}$ photoluminescence experiments. (7) At lower $I_{X}$, Maxwellian DF is a good approximation as shown in curves(a) and $(b)$. Curves for $I_{x}=I_{0}$ were analyzed for various $\tau$ and the carrier temperatures obtained from such fits are plotted as a function of time delay $\tau$ in Fig. 8. The points corresponding to curves (b) and (c) in Fig. 7 are also shown.

There are two major points of interest in Fig. 8. First of all, one can ask the question if $T_{C}(t)$ can be fit to the theory of energy loss rates (Section II $C$ ). Assuming that all the excess energy $(\approx 120 \mathrm{meV}$ ) of photoexcited carriers is effective in heating the carriers (i.e., the initial temperature $\mathrm{T}_{0}=465 \mathrm{~K}$ ) Leheny et al. (13) obtained a reasonably good fit to the data as shown by the lower solid curve in Fig. 8. The energy loss rate used for this fit was approximately $1 / 2$ the values shown in Fig. 4; hence we have 


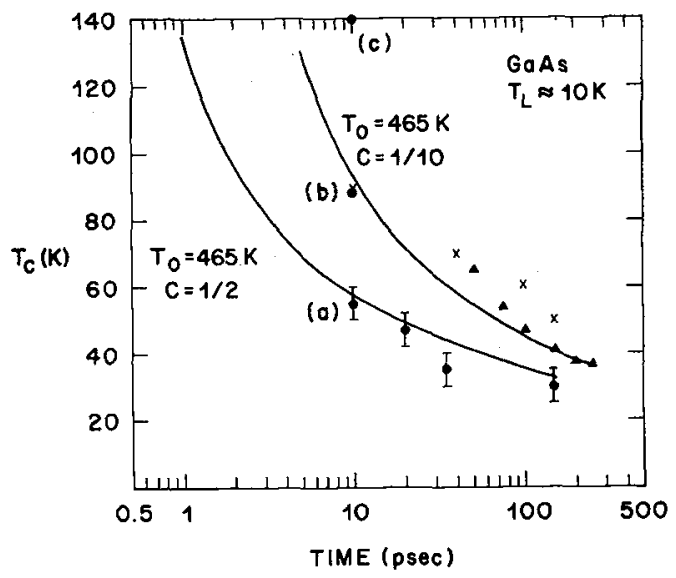

Fig. 8 The measured variation of $T_{C}$ with time delay; circles (Leheny et al., Ref. 13) triangles (von der Linde and Lambrich, Ref. 14) crosses (Tanaka et al., Ref. 3l). The points (a), (b) and (c) correspond to the three curves in Fig. 7. Theoretical curves for $T_{0}=465 \mathrm{~K}$ and $\mathrm{C}=1 / 2$ and $1 / 10$ are also shown.

labelled the curve $T_{0}=465 \mathrm{~K}, C=1 / 2$. The effect of reducing $\mathrm{T}_{0}$ is negligible for the time delays > 10 psec (see Fig. 5). One can therefore conclude that the observed energy relaxation rate is somewhat slower than the values calculated in Section II (Fig. 4). The second major point of interest is that the measured temperature at a fixed time delay increases with increasing $I_{X}$, showing that the energy loss rate to the lattice decreases at higher excitation densities. An explanation of this reduction in terms of the screening of. electron-phonon interaction was suggested by Leheny et al. (13) Assuming a further reduction of $\mathrm{C}$ by a factor of five (i.e., $\mathrm{c}=$ 1/10), the calculated curve passes close to the measured point (b). Effects of the screening of the electron-phonon interaction were calculated by Ehrenreich ${ }^{(28)}$ in 1959. The experiments described above provide the first measurement of this effect and demonstrate the importance of picosecond lasers in investigating carrier relaxation in semiconductors.

Results similar to those described above were also obtained by von der Linde and Lambrich, (14) who used two photon absorption of 25 psec pulses from a YAG laser to excite GaAs and a delayed, frequency-tunable parametric oscillator to measure the resulting changes in the transmission. Their results for $T_{C}$ vs time delay are also represented in Fig. 8. We see that their results fit the $\mathrm{C}=10$ curve better, suggesting that screening effects were important in these measurements. von der Linde et al. (29) have also investigated the lifetime of photoexcited nonequilibrium phonons by using similar techniques. 
Luminescence measurements have played an important role in investigating hot carrier relaxation in $\mathrm{CW}$ experiments. $(4,5)$ with the development of streak cameras, optical shutters and up conversion techniques, (30) luminescence studies on picosecond timescales are possible. Some luminescence studies have already been reported in GaAs. $(31,32,33)$ Tanaka et al. (31) have measured the time resolved luminescence spectra of GaAs excited by 20 psec pulses from a frequency-doubled YAG laser by using a $\mathrm{CS}_{2}$ optical kerr shutter. Their spectra at various time delays are shown in Fig. 9. These spectra

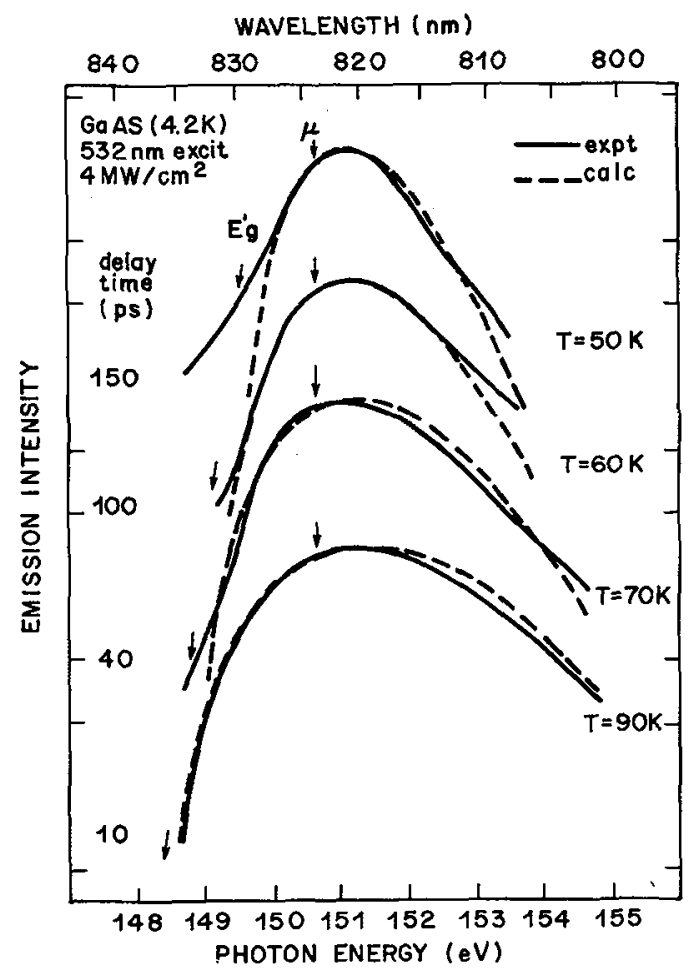

Fig. 9 Luminescence spectra of photoexcited GaAs at various time delays (from Tanaka et al., Ref. 31).

were fitted by using an analysis very similar to that described above for absorption measurements. These fits, along with the values of $E_{g}^{\prime}, \mu$ and $T_{C}$ obtained from the fits are also shown in Fig. 9. We have plotted these $\mathfrak{T}_{C}$ in Fig. 8; we note that these points also fit the $C=1 / 10$ curve reasonably well, suggesting that screening effects were important in these measurements. It is also interesting to note that $E_{g}^{\prime}$ approaches $E_{g}$ with increasing time delay; thus the temperature and 
density of photoexcited plasma in GaAs decrease with time. Precisely the same effects were observed on a much longer ( nsec) timescale in photoexcited Si by Shah and Dayem. (34)

Another interesting application of picosecond Iasers is to generate and investigate very high density plasma in semiconductors. Damen et al. (17) have recently produced densities in excess of $10^{20} \mathrm{~cm}^{-3}$ in a GaAs platelet sandwiched between two dielectric mirrors. Laser action at wavelengths as short as $7700 \AA$ was observed, and the time evolution of this laser action was investigated. Many complex physical phenomena determine the response of semiconductors under such high excitation densities. One phenomenon relevant to the discussion here is the intervalley relaxation between $I$ and $L$ or $x$ valleys. The densities excited here are high enough that electrons occupy not only the $\Gamma$ valley but also the $L$ and possibly $X$ valleys. Understanding the response of the system can provide information about such intervalley transitions.

These picosecond techniques can be applied to investigate hot carrier relaxation processes in other semiconductors. Results on case have been reported. (35) Hot carrier relaxation in GaAs-AlGaAs multiple quantum wells have been investigated recently ${ }^{(15)}$. It is clear that many interesting experiments will be forthcoming in this field.

In the experiments described above, the picosecond pulses were used to heat the carriers as well as to probe the carrier DF following such photoinduced heating. Picosecond techniques can also be used to probe the carrier relaxation and transport in the presence of an applied electric field. We have already mentioned the experiment by Shank et al. (16) in which the velocity overshoot phenomenon in GaAs was investigated. In these experiments, Shank et al. (16) made use of the fact that the application of an electric field changes the absorption spectrum of GaAs because of the FranzKeldysh effect. (36) when a low density of carriers is injected by a subpicosecond laser, the propagation of these carriers creates an opposing field and results in a change in absorption $(\Delta \alpha)$ due to a reduction in the Franz-Keldysh effect. The transport dynamics are obtained by measuring the time evolution of $\Delta \alpha$ by the pump and probe technique and comparing it with a calculated curve using the electron velocity $v_{e}(t)$ as an adjustable parameter. Their results, shown in Fig. 10, imply large initial electron velocities followed by relaxation to velocities approximating the electron saturated velocity. These measurements provide the first evidence for the velocity overshoot phenomenon predicted by Maloney and Frey. 

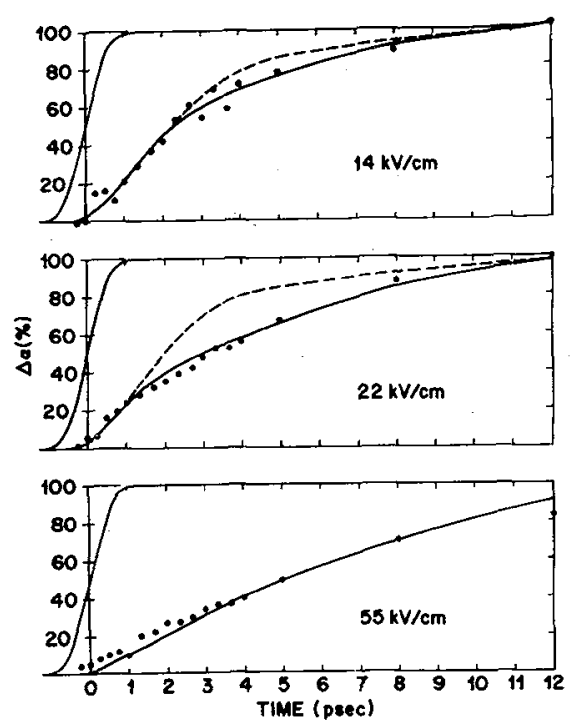

Fig. 10 optical absorption change $\Delta \alpha$ vs. time delay at three applied electric fields. The individual points are experimental. For $14 \mathrm{kV} /$ $\mathrm{cm}$ the solid line is a calculated curve using two constant velocity regimes; $v_{e}=3.0 \times 10^{7} \mathrm{~cm} / \mathrm{sec}$ $\left(t<2.4\right.$ psec) and $v_{e}=1.0 \times 10^{7} \mathrm{~cm} /$ sec $(t>2.4 \mathrm{psec})$. For $22 \mathrm{kV} / \mathrm{cm}$ the solid curve is calculated with $\mathrm{v}_{e}=$ $4.4 \times 10^{7} \mathrm{~cm} / \mathrm{sec}(t<1.1 \mathrm{psec})$ and $v_{e}=1.2 \times 10^{7} \mathrm{~cm} / \mathrm{sec}(t>1.1 \mathrm{psec})$. The dashed curves show the continuation of $\Delta \alpha(t)$ if $v_{e}$ remains constant. For $55 \mathrm{kV} / \mathrm{cm}$ the solid curve is calculated with $v_{e}-1.3 \times 10^{7}$ $\mathrm{cm} / \mathrm{sec}$ for all $\mathrm{t}$. The curve at the far left of each trace shows the instrumental response (from Shank et al., Ref. 16).

We conclude our discussion of experimental results by discussing a recent experiment by Degani et al., (38) in which the velocity field characteristics for photoexcited minority electrons in

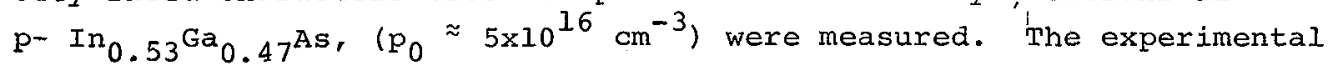
structure was $80 \mu \mathrm{m}$ wide and had a $120 \mu \mathrm{m}$ source-drain separation. The sample was mounted as a part of $50 \mathrm{ohm}$ strip line circuit, and was weakly photoexcited by $\sim 10$ psec laser pulses a distance a from the drain. The photoresponse of the device was measured with a sampling scope and averaged with a multichannel analyser. For a given applied field, the drift velocity of photoexcited electrons was determined from the slope of the transit length (d) vs pulse widths of photo response. The drift velocities deduced in this manner are plotted in Fig. 11 as a function of applied electric field and compared with the calculation of Littlejohn et al. (39) for the majority carriers in the $\mathrm{n}$-type In $0.53^{\mathrm{Ga}_{0}} .47^{\mathrm{As}}$. We see that the drift velocity of photoexcited electrons continues to increase monotonically with applied field (i.e., exhibits no negative differential mobility) and reaches values as high as $2.6 \times 10^{7} \mathrm{~cm} / \mathrm{sec}$ at $7.5 \mathrm{kV} / \mathrm{cm}$. This is an interesting result from the point of view of making fast devices. Recent photoluminescence study of $\mathrm{n}$ - and p-type $\operatorname{In}_{0.53} \mathrm{Ga}_{0.47^{\text {As }}}$ shows $(40)$ that p-type samples show substantially smaller photoinduced heating of carriers than $n$-type and undoped samples. These luminescence results can be interpreted in terms of electron-hole interactions and strong hole-lattice coupling. The absence of negative differential mobility of photoexcited minority electrons in the high field measurements 


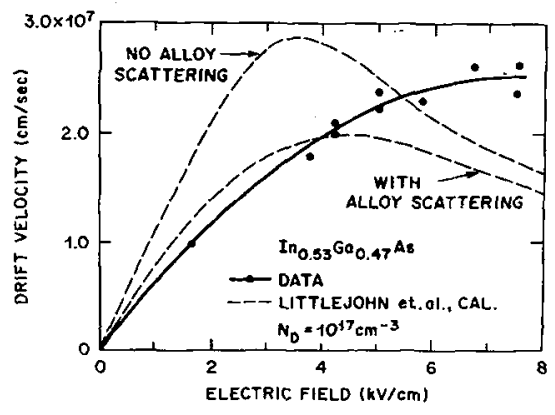

Fig. 11 Electron drift velocity as a function of applied electric field for p-type $\operatorname{In}_{0 .}{ }_{3} \mathrm{Ga}_{0} .47^{\mathrm{As}}$. The bold line, drawn through the data points, can be compared to the velocity-field calculation of Littlejohn et al. (Ref. 39) for n-type Ino. 53 Ga0.47As with $\mathrm{N}_{\mathrm{D}}=$ $1017 \mathrm{~cm}^{-3}$. (From Degani et al. Ref. 38).

discussed above was also attributed ${ }^{(39)}$ to reduced heating of electrons, and hence reduced probability of electron transfer to upper valleys, because of the presence of holes. The experiments by shank et al. (16) and the one just discussed, illustrate the usefulness of combining transport measurements with picosecond optical techniques.

IV. Conclusions

Optical techniques are extremely useful in the investigation of hot carrier relaxation in semiconductors in two distinct ways. First, they provide a means of determining the carrier distribution function under various experimental conditions. Second, photoexcitation provides a means of heating the carriers and thus facilitates the investigation of many parameters involved in hot carrier relaxation. Availability of picosecond and subpicosecond pulses in recent years has added an exciting new dimension in the study of hot carrier relaxation in semiconductors by optical techniques. We have discussed some basic theoretical considerations essential in understanding such picosecond relaxation studies. It is clear that many challenging opportunities exist for theorists in this area. The calculation of the time evolution of the distribution function of photoexcited carriers on subpicosecond timescales is one example. We have reviewed some recent experimental work which illustrates how direct information about hot carrier relaxation can be obtained by using picosecond optical pulses. Experiments using pump-probe technique, luminescence technique as well as experiments combining transport and picosecond techniques have been performed. Very high density ( $>10^{20} \mathrm{~cm}^{-3}$ ) plasma has been created and investigated using picosecond lasers. These experiments have enabled a direct measurement of carrier energy loss rates, demonstrated the importance of screening and provided evidence for the velocity overshoot phenomenon predicted previously. These experiments have also shown that photoexcited carriers reach a Maxwellian distribution function (with $T_{C}>T_{L_{L}}$ ) in less than 10 psec. The region below 10 psec (to $\approx 0.2$ psec) is now accessible 
and should yield more information about various relaxation processes. Application of picosecond pulses to investigate hot carrier relaxation promises to be an active and fxuitful field of research in coming years.

Acknowledgment

I would like to thank R. F. Leheny for many useful discussions on high density photoexcited plasma and hot carrier relaxation in semiconductors.

\section{References}

1. E. M. Conwell, Solid State Physics, Supplement 9 (Edited by F. Seitz, D. Turnbull and H. Ehrenreich) Academic Press, New York 1967.

2. G. Bauer, Springer Tracts in Modern Physics, Editor, G. Höhler, Springer-Verlag, Berlin (1974) Volume 74, p. 1 .

3. G. Bauer, Solid State Electronics 21, (1978).

4. Jagdeep Shah, Solid State Electronics 21, 43 (1978).

5. R. G. Ulbrich, Solid State Electronics 21, 51 (1978).

6. Jagdeep Shah and J. C. V. Mattos, Proceedings of the Third International Conference on Light scattering in Solids, Campinas, Brazil 1975. Edited by M. Balkanski, R. C. C. Leite and S. P. S. Porto, Flammarion, Paris (1975), p. 145.

7. Jagdeep Shah, Phys. Rev. B10, 3697 (1974).

8. Second International Conference on Hot Carriers in semiconductors, Denton, Texas 1977. Proceedings of this conference are published as volume 21 of Solid State Electronics (1978). This and the next reference contain many articles describing the use of optical techniques for generating and probing hot carriers.

9. Physics of Nonlinear Transport, Edited by D. K. Ferry, J. R. Barker and C. Jacobani, NATO Advanced Study Institute Series B: Physics, vol. 52 Plenum Press, New York 1980.

10. See, for example, "Picosecond Phenomena II" Proceedings of the Second International Conference on Picosecond Phenomena, Edited by R. Hochstrasser, W. Kaiser and C. V. Shank, Springer-Verlag, Berlin 1981 .

11. Earlier work on Germanium has been reviewed recently by A. Smirl, p. 367 , Ref. 9 .

12. C. V. Shank, R. L. Fork, R. F. Leheny and Jagdeep Shah, Phys. Rev. Letters $\underline{42}, 112$ (1979).

13. R. F. Leheny, Jagdeep Shah, R. L. Fork, C. V. Shank and A. Migus, Solid State Communications 31, 809 (1979). 
14. D. von der Linde and R. Lambrich, Phys. Rev. Letters $\underline{42}, 1090$ (1978).

15. C. V. Shank, R. L. Fork, B. I. Greene, C. Weisbuch and A. Gossard to be published. See also the paper by C. Weisbuch, C. V. Shank, R. L. Fork, B. I. Greene, A. C. Gossard and W. Wiegmann, at this conference.

16. C. V. Shank, R. L. Fork, B. I. Greene, F. K. Reinhart and R. A. Logan, Applied Physics Letters 38, 104 (1981).

17. T. C. Damen, M. A. Duguay, Jagdeep Shah, J. Stone, J. M. Wiesenfeld and R. A. Logan, to be published in Applied Physics Letters (1981).

18. C. J. Hearn, p. 343, Ref. 9, has recently discussed theoretical concepts of photoexcited hot carriers. See also Ref. 4 and Ref.5

19. R. L. Fork, B. I. Green and C. V. Shank, Applied Physics Letters 38, 671 (198I).

20. Jagdeep Shah, R. F. Leheny and W. Wiegmann, Phys. Rev. B15, 1577 (1977).

21. W. F. Brinkman and T: M. Rice, Phys. Rev B7, 1508 (1973).

22. For recent reviews, see T. M. Rice, and J. C. Hensel, T. G. Phillips, and G. A. Thomas in Solid State Physics, Vol.32, edited by F. Seitz, D. Turnbull and H. Ehrenreich, Academic Press, New York 1977 .

23. J. Shah, R. F. Leheny, W. R. Harding and D. R. Wight, Phys. Rev. Letters 38,1164 (1977).

24. R. F. Leheny and J. Shah, Phys. Rev. Letters 37, 871 (1976).

25. For a review of scattering rates for holes, see $J$. Wiley, in Semiconductors and Semimetal, edited by R. K. Willardson and and A. C. Beer (Academic Press, New York, 1975) Vol. 10, p. 91.

26. E. Goebel and 0 . Hildebrand, Phys. Stat. Solidi (b) 88,645 (1978).

27. E. P. Ippen and C. V. Shank, in "Picosecond Phenomena," Proceedings of the First International Conference on Picosecond Phenomena edited by C. V. Shank, E. P. Ippen and S. L. Shapiro published by Springer Verlag, Berlin 1978, p. 103.

28. H. Ehrenreich, J. Phys. Chem. Solids 8,130 (1959).

29. D. von der Linde, J. Kuhl, H. Klingenberg, Phys. Rev. Letters 44 , 1505 (1980).

30. H. Mahr and M. C. Hirsch, Optics Communications 13, 96 (1975).

31. S. Tanaka, H. Kobayashi, H. Saito and H. Shionoya, J. Phys. Soc. Japan 49, 1051 (1980).

32. W. Graudszus and E. Goebel, at this conference. 
33. R. J. Seymour, M. R. Junnarkar and R. R. Alfano, Bull Am. Phys. Soc. 26,458 (1981).

34. Jagdeep Shah and A. H. Dayem, Phys. Rev. Letters 37, 861 (1976).

35. T. Daly and H. Mahr, Solid State Communications 25, 323 (1978).

36. W. Franz, Z. Naturforch. 13,484 (1958) and L. V. Keldysh, Soviet Physics JETP 6, 763 (1958).

37. T. J. Maloney and J. Frey, J. Applied Physics 48, 781 (1977).

38. J. Degani, R. F. Leheny, R. E. Nahory and J. Heritage, to be published.

39. M. A. Littlejohn, J. R. Hauser, T. H. Glisson, D. K. Ferry and J. W. Harrison, Solid State Electronics 2l, 107 (1978).

40. Jagdeep Shah, R. E. Nahory, R. F. Leheny, J. Degani and A. E. DiGiovanni, to be published. 